\title{
Los juzgados constitucionales para una protección eficaz de los derechos constitucionales en el Distrito Judicial de Áncash
}

\author{
The constitutional court for an effective protection of constitutional rights in \\ the Judicial District of Ancash \\ Luis Robles Trejo ${ }^{1}$, Elmer Robles Blácido ${ }^{1}$, Pepe Melgarejo Barreto ${ }^{1}$ y \\ Wilfredo Montañez Avendaño ${ }^{1}$
}

\section{RESUMEN}

Este estudio determina la viabilidad e importancia de la creación o implementación de los juzgados especializados en materia constitucional para una protección adecuada y oportuna de los derechos constitucionales en el Distrito Judicial de Áncash. Se trata de un estudio retrospectivo, longitudinal, correlacional, cuantitativo, diseño no experimental, desarrollado en el ámbito de la jurisdicción de la Corte Superior de Huaraz, durante los años 2010-2011. La muestra estuvo constituida por 120 abogados litigantes pertenecientes al Colegio de Abogados de Áncash y 33 magistrados del Distrito Judicial de Áncash, seleccionada de acuerdo a criterios de inclusión y exclusión, utilizando como instrumentos de recolección de datos la encuesta y la ficha de análisis de contenido respectivamente. De la aplicación de los IRD se obtuvo los siguientes resultados: disconformidad con el actuar de los magistrados (83\%); no actúan como jueces constitucionales (92\%); no respetan los principios que rigen los procesos constitucionales (95\%); no respeto de la tramitación preferente (92\%); trasgresión de los principios de los procesos constitucionales de la libertad (96\%); trasgresión de los principios que rigen los procedimientos constitucionales (83\%); no aplicación de la prueba de oficio (97\%); violación de los plazos procesales (92\%); sobre la responsabilidad del agresor $(83 \%)$; jueces constitucionales de Sala (92\%); labor de no corrección de la Sala (84\%); Mecanismos no eficaces (92\%). Se concluye que los derechos constitucionales no están siendo protegidos adecuada, oportuna y efectivamente, y esto se debe a la falta de especialización de los magistrados, quienes no aplican los principios que orientan los procesos constitucionales y terminan desnaturalizándolos, por lo que la administración de justicia constitucional en el Distrito Judicial de Áncash es deficiente; y esto justifica la creación o implementación de los juzgados constitucionales.

Palabras clave: juzgados constitucionales; especialización; derechos constitucionales; protección; eficacia; garantías.

\begin{abstract}
This research determines the feasibility and importance of the creation or implementation of specialized courts in constitutional matters for adequate and timely protection of constitutional rights in the Judicial District of Ancash. This is a retrospective, longitudinal, correlational, quantitative, non-experimental design, developed in the area of jurisdiction of the Superior Court of Huaraz, in 2010-2011. The sample con-
\end{abstract}

1 Universidad Nacional «Santiago Antúnez de Mayolo». Huaraz, Perú. 
sisted of 120 trial lawyers belonging to the State Bar of Ancash and 33 judges of the Judicial District of Ancash, selected according to inclusion and exclusion criteria, using as a data collection instrument and the survey and content analysis card respectively. In the application of IRD following results were obtained: Disagreed with the actions of judges $(83 \%)$ do not act as constitutional judges (92\%) do not respect the principles governing constitutional processes $(95 \%)$, lack of respect for preferential processing (92\%), violation of the principles of constitutional liberty processes $(96 \%)$, violation of the principles governing constitutional procedures $(83 \%)$, non-enforcement of trade test $(97 \%)$, violation of procedural deadlines $(92 \%)$, on the responsibility of the offender (83\%) Sala constitutional judges $(92 \%)$ work no correction of the Chamber (84\%); mechanisms ineffective (92\%). Concluding that constitutional rights are not being protected adequately, timely and effectively, and this is due to the lack of specialization of judges who do not apply the guiding principles of constitutional processes and end denaturing, so the administration of justice Constitution in the Judicial District of Ancash is poor, and this justifies the creation or implementation of constitutional courts.

Keywords: constitutional courts; expertise; constitutional rights; protection; efficiency; warranties.

\section{ICHIKLLACHAW}

Kay musyay ashipakuyqa husgadukunapa kawayninta alli naanintam alliq riqinapaq willakun, hinaman allilla washaynin kananpaqmi riman, kunstitusiyunalkunapa dirichunkunata Anqash hudisyal distritunchaw kanqantam. Kay musyapakuyqa ritruspiktibum, lunhitudinal, kurilasiyunal, kwantitatibu, mana ikspirimintalmi kayan Waraspa Kurti Supiryurchaw 2010wan 2011 watakunachaw musyapakushqam. Tsay 120 abugadukunawanmi rurakashqa, paykuna llapan kayan Anqashpa kulihyun abugadukunam, hinaman 33 Anqashpa distritu hudisyal mahistradunkunam, paykuna yaykuywan yarquy kaqwanmi akrakashqa, tsayta ayllunapaq inkuwista nishqantam rurakashqa, hinaman huwicha nishqantapis wanakashqam. IRD rurashqanpiq kaykunam yarqushqa: mahistradukuna mana alli rurayninkuna (83\%), huwis kunstitusiyunalnaw mana rurayanqan (92\%), prusisu kunstitusiyunalta mana qullanankuna (95\%), tramitasiyunta mana kaasuq (92\%), libirtapa prinsipyunta allqutsaq (96\%), naani aywaynin prinsipyunta allqutsaq (83\%), huwisyu prubibanta mana kaasuq (97\%), prusisalkunapa plaasunkuna mana kaasukuqkuna (92\%), salapa alli kayninta uryaynin (84\%), mana alliq kay mikanismukuna (92\%). Tsay llapanta ichik shimillachaw nishwan manam allitsu washayninkuna kaykan, kunstitusiyunal dirichukuna manam alliqllaqa rikakantsu, imaraykurmi tsaynaw, mahistradukunapam yachayninkuna pishin, tsaymi prinsipyupa naaninta alliq rurayantsu, kaynam mana alli ushan. Tsayraykurmi Anqashpa distritu hudisyalnin pasaypa mana hustisyata qatiyan. Tsayraykurmi kay mushuq husgadukuna yurinman.

Pushaq shimikuna: kunstitusiyunal husgadukuna; ispisyalisasiyun; kunstitusiyunal dirichukuna; washaynin alli kaynin garantiyakuna.

\section{INTRODUCCIÓN}

El neoconstitucionalismo se ha convertido en el paradigma para poder explicar los fenómenos jurídicos; en ese sentido qué duda cabe hoy, que el Derecho se ha constitu- 
cionalizado, a tal punto que la constitución se irradia y extiende a todo el ordenamiento jurídico y a todas las disciplinas jurídicas. Este nuevo paradigma es fruto de una profunda transformación interna del paradigma positivista, es decir, una transformación que afecta no solo a la naturaleza del derecho en la medida en que «la positivización que resulta de los derechos fundamentales como límites y vínculos sustanciales a la legislación positiva», sino también, conlleva, una nueva dimensión acerca del principio de legalidad o legalidad sustancial, el cual permite perfeccionar el clásico principio de legalidad formal o mera legalidad antes paradigmático, lo cual supone, que el sometimiento a la ley, no se gesta única y exclusivamente a vínculos formales, y ahí radica la esperanzadora novedad, sino que a vínculos sustanciales, derivados de los principios y derechos fundamentales consignados en la Constitución, lo cual influye en la estructura del Estado Constitucional.

Por otro lado, para comprender cómo la Constitución actúa en su calidad de norma vinculante y con eficacia jurídica inmediata, hay que distinguir la naturaleza de los preceptos contenidos en ella. Al respecto, es posible distinguir disposiciones constitucionales que contienen principios rectores de política social y económica por un lado y otros preceptos que realizan una remisión normativa a la ley, pero de ambos es necesario concluir que en ningún caso la Constitución goza de normas programáticas, ello fluye del hecho de que la Carta Fundamental establece un plexo valórico de principios, derechos y deberes todos los cuales conllevan sus respectivos mecanismos garantes.

Así mismo, tenemos a la Justicia Constitucional que requiere ser analizada no solo desde un plano teórico, sino fundamentalmente desde la práctica del funcionamiento de los jueces y órganos jurisdiccionales. Es decir, desde el impacto real de sus sentencias en el ámbito social y de las expectativas de los ciudadanos, en el marco del Estado social y democrático de Derecho, en tanto garantes y defensores de la Constitución. Desde la normatividad constitucional peruana, resulta jurídicamente sostenible que el Estado no satisface su deber de garantía de los derechos fundamentales (artículo 44 de la Constitución), a través de la mera declaración de la conculcación de un derecho fundamental por el juez constitucional; sino que se debe ordenar el aparato estatal, dotándolo de los mecanismos necesarios para garantizar el efectivo goce de los mismos (artículo 1 de la Constitución), que supone la plena reparación a través del cumplimiento de dicho pronunciamiento.

\section{MATERIALES Y MÉTODOS}

Se trata de un estudio retrospectivo, longitudinal, correlacional, cuantitativo, diseño no experimental, debido a que careció de manipulación la variable independiente; además no contó con grupo de control; su finalidad fue analizar el hecho jurídico identificado en el problema después de su ocurrencia.

Los métodos utilizados fueron: análisis-síntesis, para poder descomponer el problema en sus partes y determinar sus características; así como, a partir de dichos elementos entender el comportamiento como unidad de análisis. Exegético, para el análisis sistemático de la normatividad y para explicar cada parte del conjunto del texto normativo, tratando de definir cada concepto y enunciar el significado normativo de cada disposición. Hermenéutico, para interpretar los textos dentro de un sistema de pensamiento y encontrar su verdadero significado, a partir de los principios y conceptos generales. 
Argumentación jurídica, el cual permitió justificar las teorías jurídicas, demostrar lógicamente por medio de un razonamiento formulado con el propósito de conseguir la aceptación o rechazo de una teoría determinada. Método matemático, la investigación por su naturaleza mixta, trabajó con datos empíricos que requirieron de representación numérica, en consecuencia se empleó las matemáticas para poder representar dichos datos o información -cuantificación numérica-, Método estadístico, para la interpretación y representación en tablas y gráficos estadísticos de los datos obtenidos.

Las técnicas, utilizadas para la recolección de información fueron: Análisis de Registro Documental. Esta técnica estuvo en función del análisis doctrinario y teórico de las diversas obras, así como de la jurisprudencia emitida por el Tribunal Constitucional. Encuesta para recoger la opinión de los abogados y operadores jurídicos. Técnica de análisis de contenidos para el estudio de la jurisprudencia y las resoluciones judiciales.

\section{RESULTADOS}

Cuadro resumen de la carga procesal y producción judicial en trámite por órgano jurisdiccional y especialidad: Distrito Judicial de Áncash, periodo 2009-2011, en la tabla 1.

Tabla 1. Carga procesal y producción judicial

\begin{tabular}{|c|c|c|c|c|c|c|}
\hline \multicolumn{7}{|c|}{ Carga Procesal } \\
\hline \multirow{2}{*}{$\begin{array}{c}\text { ÓRGANO/ } \\
\text { ESPECIALIDAD }\end{array}$} & \multicolumn{2}{|c|}{2009} & \multicolumn{2}{|c|}{2010} & \multicolumn{2}{|c|}{2011} \\
\hline & TOTAL & $\begin{array}{c}\text { PROD. } \\
\text { JUDICIAL }\end{array}$ & TOTAL & $\begin{array}{c}\text { PROD. } \\
\text { JUDICIAL }\end{array}$ & TOTAL & $\begin{array}{c}\text { PROD. } \\
\text { JUDICIAL }\end{array}$ \\
\hline \multicolumn{7}{|c|}{ Sala Superior Especializada } \\
\hline Civil & 1,464 & 1,093 & 699 & 1,641 & 2,453 & 2,094 \\
\hline Penal & 3,910 & 2,962 & 1,243 & 2,218 & 4,760 & 2,845 \\
\hline \multicolumn{7}{|c|}{ Juzgado Especializado o Mixto } \\
\hline Civil & 3,514 & 1,681 & 1,438 & 1,241 & 2,616 & 787 \\
\hline Familia & 5,287 & 2,416 & 7,258 & 2,150 & 5,228 & 1,912 \\
\hline Mixto & 17,770 & 5,429 & 12,876 & 6,666 & 19,068 & 6,338 \\
\hline Penal & 14,511 & 6,541 & 6,539 & 2,868 & 10,629 & 4,895 \\
\hline \multicolumn{7}{|c|}{ Juzgado de Paz Letrado } \\
\hline Juzgado de Paz & & & & & & \\
\hline Letrado & 13,762 & 4,798 & 6,209 & 5,274 & 15,470 & 5,710 \\
\hline Total & 60,218 & 24,920 & 36,262 & 22,058 & 60,224 & 24,581 \\
\hline
\end{tabular}

Resultados de la encuesta aplicada a los abogados litigantes de la corte superior de Áncash sobre la administracion de justicia constitucional y la labor de los jueces ordinarios como jueces constitucionales

1. Percepción de los abogados sobre el actuar de los jueces de la Corte Superior de Áncash, como jueces constitucionales, cuando han resuelto los procesos constitucionales. 
Tabla 2. Labor de los magistrados como jueces constitucionales

\begin{tabular}{lcc}
\hline \multicolumn{1}{c}{ RESPUESTAS } & $\mathrm{f}$ & $\mathrm{f} \%$ \\
\hline Sí & 10 & 8 \\
No & 110 & 92 \\
Total & 120 & 100 \\
\hline
\end{tabular}

2. Respeto de los principios que rigen los procesos constitucionales por parte de los jueces de la Corte Superior de Áncash.

Tabla 3. Respeto de los principios que rigen los procesos constitucionales

\begin{tabular}{lcc}
\hline \multicolumn{1}{c}{ RESPUESTAS } & $\mathrm{F}$ & $\mathrm{f} \%$ \\
\hline Sí & 5 & 4 \\
No & 115 & 96 \\
Total & 120 & 100 \\
\hline
\end{tabular}

3. Labor de los jueces de primera instancia de la Corte Superior de Áncash sobre si han respetado lo prescrito en el artículo $13^{\circ}$ del Código Procesal Constitucional referido a la tramitación preferente de los procesos constitucionales.

Tabla 4. Tramitación preferente de los procesos constitucionales

\begin{tabular}{lcc}
\hline \multicolumn{1}{c}{ RESPUESTAS } & $\mathrm{F}$ & $\mathrm{f} \%$ \\
\hline Totalmente en desacuerdo & 0 & 0 \\
De acuerdo & 10 & 8 \\
Desacuerdo & 65 & 54 \\
Totalmente en desacuerdo & 45 & 38 \\
Total & 120 & 100 \\
\hline
\end{tabular}

4. Labor de los jueces de primera instancia de la Corte Superior de Áncash acerca del respeto de los plazos procesales fijados por el Código Procesal Constitucional para resolver un proceso constitucional.

Tabla 5. Plazos procesales

\begin{tabular}{lcc}
\hline \multicolumn{1}{c}{ RESPUESTAS } & $\mathrm{F}$ & $\mathrm{f} \%$ \\
\hline Siempre & 0 & 0 \\
A Veces & 10 & 8 \\
Nunca & 110 & 92 \\
Total & 120 & 100 \\
\hline
\end{tabular}

5. Labor de los magistrados de la Sala de la Corte Superior de Áncash que vienen actuando como jueces constitucionales. 
Tabla 6. Labor de los magistrados de Sala como jueces constitucionales

\begin{tabular}{lcc}
\hline \multicolumn{1}{c}{ RESPUESTAS } & $\mathrm{F}$ & $\mathrm{f} \%$ \\
\hline Totalmente de acuerdo & 0 & 0 \\
De acuerdo & 10 & 8 \\
Desacuerdo & 65 & 54 \\
Totalmente en desacuerdo & 45 & 38 \\
Total & 120 & 100 \\
\hline
\end{tabular}

6. Labor de los magistrados de la Sala de la Corte Superior de Áncash, sobre corrección de errores cometidos por los jueces de primera instancia, en referencia a los procesos constitucionales.

Tabla 7. Corrección por parte de los magistrados de sala

\begin{tabular}{lcc}
\hline \multicolumn{1}{c}{ RESPUESTAS } & $\mathrm{f}$ & $\mathrm{f} \%$ \\
\hline Siempre & 0 & 0 \\
A Veces & 18 & 15 \\
Nunca & 102 & 85 \\
Total & 120 & 100 \\
\hline
\end{tabular}

7. Opinión de los abogados sobre la eficacia de los procesos constituciones, en la Corte Superior de Áncash, como mecanismos de protección real y efectiva de los derechos constitucionales.

Tabla 8. Eficacia de los procesos constitucionales en la Corte Superior de Áncash

\begin{tabular}{lcc}
\hline \multicolumn{1}{c}{ RESPUESTAS } & $\mathrm{f}$ & $\mathrm{f} \%$ \\
\hline Sí & 10 & 8 \\
No & 110 & 92 \\
Total & 120 & 100 \\
\hline
\end{tabular}

\section{DISCUSIÓN}

\section{Discusión empírica}

Los resultados de la tabla 1, expresan la carga procesal y producción judicial tramitada por órgano jurisdiccional y especialidad en el Distrito Judicial de Áncash, durante el periodo 2009-2011, donde se puede observar la preeminencia de los procesos civiles sobre los penales, y la alta carga procesal que despachan los órganos de esta sede jurisdiccional.

Los resultados de la tabla 2 expresan que los abogados están disconformes con el actuar de los magistrados de la Corte Superior de Áncash cuando han administrado justicia constitucional a través de los procesos constitucionales que tuvieron a su cargo al resolver, es decir, existe una percepción negativa por parte de los abogados respecto al actuar de los magistrados no solo en los procesos constitucionales sino en general 
respecto al actuar en todos los procesos judiciales, y esta disconformidad se manifiesta también en los referéndums que realiza el Colegio de Abogados de Áncash. Así mismo, respecto al actuar y conducta funcional de los magistrados de esta jurisdicción, la gran mayoría de ellos salen desaprobados, según los ítems que se les evalúa que son celeridad procesal, fundamentación de resoluciones, trato a los abogados y justiciables.

Los resultados de la tabla 3 expresan que los jueces ordinarios cuando resuelven los procesos constitucionales no respetan los principios que rigen los procesos constitucionales, en consecuencia no estarían actuando como jueces constitucionales que es el rol que les toca desempeñar, y este hecho trae graves consecuencias en la protección de los derechos constitucionales; ya que siendo los principios constitucionales las ideas rectoras del proceso constitucional y al no ser tomadas en cuenta por el juez ordinario que conoce la causa, lo que se produce es la desnaturalización del proceso constitucional y al final termina siendo tramitado como un proceso ordinario más, vulnerando la tutela de urgencia que amerita estos procesos constitucionales. El hecho que los jueces ordinarios no actúen como verdaderos jueces constitucionales, conlleva a que no se estén cumpliendo los fines de los procesos constitucionales establecidos en el artículo II del Título Preliminar del Código Procesal Constitucional, como son garantizar el principio de supremacía constitucional ni la vigencia real y efectiva de los derechos constitucionales, por tanto no se están comportando como guardianes de las constituciones en términos de Carl Schmitt, sino que están actuando como jueces mármoles, jueces pétreos, jueces robóticos en términos de Montesquieu.

Los resultados de la tabla 4 expresan que los jueces ordinarios cuando resuelven procesos constitucionales no respetan lo prescrito por el artículo $13^{\circ}$ del Código Procesal Constitucional. Este regula la tramitación preferente de los procesos constitucionales frente a los procesos ordinarios; por ello, este se convierte en uno de los puntos críticos de la justicia constitucional, ya que al no otorgarles por parte del juez una preferencia a los procesos constitucionales de la libertad. El juez está equiparando la violación de un derecho constitucional con la violación de un derecho legal, es decir, para el juez ambos tienen la misma importancia y trascendencia jurídica, hecho que es considerado por la doctrina constitucional como una aberración jurídica y esto es desconocer el carácter jurídico de la constitución, es desconocer que los jueces constitucionales están llamados a garantizar el contenido de la constitución.

Los resultados de la tabla 5 expresan que los jueces no respetan los plazos procesales establecidos en el Código Procesal Constitucional para resolver un proceso constitucional, hecho grave para la justicia constitucional, ya que cuando uno acude a la jurisdicción constitucional lo hace considerando la tutela de urgencia que caracteriza a dichos procesos. Por ello cuando los plazos no se cumplen tampoco se está dando cumplimiento a sus fines, porque el fin es la vigencia efectiva de los derechos constitucionales y no retardarla, por ello es que los justiciables no creen en la justicia ordinaria ni constitucional, porque hacen uso del adagio popular «justicia que tarda no es justicia». Por ello es que el legislador considerando la carga procesal que caracteriza al Poder Judicial estableció la tramitación preferente de los procesos constitucionales, porque lo que está en juego en un proceso constitucional es un contenido de la constitución, un derecho de relevancia constitucional, por ello la demora en el tiempo para resolverlo no tiene justificación. 
Los resultados de la tabla 6 expresan que los magistrados de la Sala o Segunda instancia al igual que los jueces de primera instancia no vienen actuando como jueces constitucionales. Su rol y labor no cambian cuando resuelven procesos constitucionales, pues están actuando sin respetar los principios que rigen los procesos constitucionales, o tienen interpretaciones mecánicas restrictivas y no dinámicas o amplias que caracterizan al juez constitucional.

Los resultados de la tabla 7 expresan que los magistrados de segunda instancia no están corrigiendo los errores que se cometen en primera instancia. Al contrario, en la mayoría de casos blindan o protegen, y esto se muestra con objetividad en los casos cuando el juez de primera instancia declara infundada o improcedente y esta resolución es apelada, la Sala en la mayoría de los casos confirma la resolución apelada, y no hace mayor análisis jurídico constitucional del caso y resuelve con los mismos argumentos de juez de primera instancia.

Los resultados de la tabla 8 expresan que los procesos constitucionales en el Distrito Judicial de Áncash, no son mecanismos o instrumentos eficaces de protección de los derechos constitucionales. Lo que implica que dichos procesos no están cumpliendo con los fines que la Constitución les otorga y consecuentemente se está dejando en el desamparo al ciudadano quien no encuentra un mecanismo idóneo, efectivo, rápido y sencillo para garantizar sus derechos.

\section{Discusión teórica}

2.1 La mutación del rol del juez desde un estado de derecho hacia un estado constitucional de derecho

La función jurisdiccional, bajo el Estado de Derecho, se somete a la ley y al principio de legalidad como únicas fuentes de legitimación. La labor judicial se fundamenta, pues, en un sistema normativo pleno, presuntivamente conocido por todos y cuya aplicación corresponde a autoridades independientes (Poder Judicial), cuya función consiste en resolver todas las controversias que se promuevan en el orden temporal con sujeción a normas preexistentes (López, 2001).

Superando la concepción legal-formalista del Estado de Derecho, el Estado Constitucional surge como producto de la difusión en Europa, tras la segunda guerra mundial, de las Constituciones rígidas y del control de constitucionalidad de las leyes ordinarias (Aguiló, 2004). Así, comparativamente, podemos afirmar que a diferencia del Estado de Derecho, el Estado Constitucional se encuentra basado en el principio de legalidad material, por lo que en este caso el papel del Derecho no se limita a determinar competencias y procedimientos, sino que, además, contempla límites materiales a la actividad normativa del poder político.

El papel de la jurisdicción se altera también radicalmente. El juez ya no es el ciego aplicador de la ley, o como se ha denominado muchas veces, boca de la ley. Esto no significa, sin embargo, que el juez deja de aplicar la ley, sino únicamente, que debe hacerlo solo si la ley es constitucionalmente válida (Carbonell, 2003).

Este constitucionalismo contemporáneo al que nos estamos refiriendo, ha sido también denominado neoconstitucionalismo, caracterizado por poseer una Constitución con fuerte contenido normativo y garantía jurisdiccional, se nutre efectivamente de estas 
dos fuentes. De la primera de estas tradiciones recoge la garantía jurisdiccional y una correlativa desconfianza ante el legislador. De la segunda tradición hereda, sin embargo, un ambicioso programa normativo que va bastante más allá de lo que exigiría la mera organización del poder mediante el establecimiento de las reglas de juego.

\subsection{El rol del poder judicial en el Estado Constitucional de Derecho}

La labor del Poder Judicial en el Estado Constitucional de Derecho, en general, y del juez, en particular, ha enrumbado un nuevo camino, toda vez que (u)no de los rasgos que mejor definen el Estado Constitucional de derecho es la orientación del Estado a la protección de los derechos al margen (o incluso por encima) de la ley: ya no eficacia de los derechos en la medida y en los términos enmarcados en la ley, sino eficacia de los derechos en la medida y en los términos establecidos en la Constitución (Gascon, 2005). Esa labor del Estado es encargada al Poder Judicial, observando la justicia al caso en concreto con respeto a los principios y preceptos constitucionales.

\subsection{Especialización en Derecho constitucional}

Consideramos que, en primer orden, es preciso que el juez constitucional seleccionado acredite un importante grado de vinculación y conocimiento frente al contenido normativo y valorativo de la Constitución, lo cual puede tener como modelo objetivo la especialización en Derecho constitucional, siendo importante que comprenda las técnicas del Derecho constitucional y las del Derecho procesal constitucional. Es decir, el conocimiento de la dogmática constitucional se transforma en un imperativo técnico básico. Si el magistrado constitucional quiere ser un buen técnico jurídico, a su vez tiene que ser un buen dogmático. No obstante, se debe procurar estándares comunes de calificación en los candidatos, que deberán tener expresión en un perfil concreto de profesional, del cual será posible predicar las siguientes competencias (habilidades, capacidades, aptitudes y destrezas) en materia constitucional, relevantes para el ejercicio de la magistratura constitucional (Álvarez, 2009).

\subsection{La valoración probatoria en los procesos constitucionales de la libertad}

La temática relativa a la probanza ha sido, desde siempre, el tema más controversial y apasionante de la estrella más fulgurante del universo del Derecho: el proceso. Inagotable fuente de estudio y debate ha bifurcado en dos aguas insolubles a los principales mentores de esta disciplina. Hasta los filósofos más ecuánimes y conciliadores se ven irremediablemente atraídos irreversiblemente como por un inmenso imán a uno u otro polo ideológico: el activismo o el garantismo.

No escapamos a esta opción de elegir uno u otro, pues ambos están en las antípodas. Lo tenemos claro. Si el ojo censor de ver el proceso tiene un iris constitucional entonces lo divisa como el último bastión de la libertad, esto es, «como claro medio de discusión entre personas que son siempre naturalmente desiguales pero que deben gozar de igualdad jurídica en el debate (Alvarado, 2007). Entonces nunca entenderemos la regulación de la prueba oficiosa. No solo porque atenta contra la imparcialidad del juez sino porque afecta la indispensable congruencia que tiene que existir entre el papel de las partes y el juez en el proceso (Montero, 2005). 
2.5 El desconocimiento de la especial naturaleza de los procesos constitucionales de la libertad

Un motel común donde se aloja el error en cualquier parte del camino es el siguiente: Colocar al proceso constitucional en el mismo hábitat en el que conviven los demás procesos peruanos. Desconocer que su carácter de proceso constitucional lo superpone al resto de procesos legales, es el principio de una cadena de despropósitos que se hace interminable.

Mientras en un proceso civil o penal, por señalar a los más recurridos, la norma sustantiva regula de manera plena los derechos y la labor del juzgador se hace más de ponderación y criterio, en el Habeas Corpus y el Amparo, la norma aplicable es incompleta, imprecisa y con claras implicancias políticas: la Constitución. Así, la labor del juzgador da un giro de $180^{\circ}$ y se convierte en escrupuloso intérprete de derechos fundamentales que solo se encuentran enumerados pero no desarrollados de manera explícita. Este cambio de roles y responsabilidades no debería llamar la atención si no fuera que con el Derecho peruano anida una verdad del tamaño de una montaña: la escasez de jueces constitucionales especializados. Esto genera una postal muy precaria para un país que se precia de garantizar los derechos fundamentales de sus ciudadanos: jueces legales (generalmente civiles y penales) son los que resuelven los procesos constitucionales más importantes como el habeas corpus y el amparo.

Creemos que esta inconsistencia va más allá de la especialidad. El juez ordinario no está premunido de las competencias para determinar no solo el derecho de fondo, sino que con el carácter residual del amparo, ahora debe resolver sobre su procedencia. El Estado le pide y exige al magistrado que haga algo para lo que no ha sido preparado: valorar la prueba constitucional. En el mejor de los escenarios, estará capacitado para aplicar la sana crítica o el criterio de conciencia a una controversia de contenido legal. La interpretación constitucional tiene otra lógica y un espectro infinitamente más amplio.

Solo así puede entenderse la degeneración de las medidas cautelares en el Amparo durante la vigencia de la Ley $\mathrm{N}^{\circ} 23506$ en que la amparización que se acrecentó desde los años finiseculares, la timidez de los fallos en procesos mediáticos y de la débil jurisprudencia producida en casi veinte años. Este riesgo no puede ser deporte de aventura para los derechos inmanentes de todo ser humano. Es a partir de la jurisprudencia del Tribunal Constitucional que se empieza un desarrollo intenso del texto constitucional no exento de excesos y gruesas sombras de ensoberbecimiento. Es a partir de estas interpretaciones, que se soportan en la doctrina extranjera, la jurisprudencia del Tribunal Constitucional Español, del Tribunal Federal Alemán y de la Suprema Corte norteamericana principalmente, que se ha dado un aparente avance en esta hermenéutica probatoria tan singular. Sin embargo, el órgano jurisdiccional más importante del país no tiene clara la filosofía jurídica a la cual se adscribe y plantilla jurisprudencias foráneas importantes pero inaplicables para nuestro país.

\section{CONCLUSIONES}

Los jueces ordinarios del Distrito Judicial de Áncash, al momento de resolver los procesos constitucionales no están actuando como jueces constitucionales y muestran 
conductas que no solo se constriñen al cumplimiento normativo, sino también al desarrollo de ciertas actitudes judiciales no compatibles con las de un juez constitucional.

El nivel de protección de los derechos constitucionales por parte de los magistrados de los juzgados penales y mixtos o civiles del Distrito Judicial de Áncash, es deficiente, lo cual se evidencia en la ineficacia de los procesos constitucionales, en la falta de cumplimiento de los plazos de los procesos, en la tramitación, en la falta de interpretación constitucional de los derechos conculcados o vulnerados, en el número mínimo de resoluciones judiciales declaradas fundadas, en la no ejecución de las sentencias estimatorias, en el desconocimiento de la doctrina constitucional contemporánea.

Que los derechos constitucionales al no ser protegidos de manera adecuada, oportuna y de forma efectiva, como consecuencia de la falta de especialización de los magistrados ordinarios, quienes no aplican los principios que orientan los procesos constitucionales y terminan desnaturalizándolos. La administración de justicia constitucional en el Distrito Judicial de Áncash resulta deficiente; y esto justifica la creación o implementación del juzgado constitucional.

El establecimiento del Estado Constitucional, y la influencia del paradigma de la constitucionalización del derecho, trae consigo los fundamentos doctrinales (supremacía de la constitución), normativos (constitución como norma jurídica) y procesales (garantía y protección de los derechos constitucionales) que sustentan o justifican la implementación o creación de los juzgados especializados en materia constitucional para una protección adecuada y oportuna - eficaz de los derechos constitucionales en el Distrito Judicial de Áncash.

\section{REFERENCIAS BIBLIOGRÁFICAS}

Aguiló, Josep. 2004. La Constitución del Estado Constitucional. Lima-Bogotá: PalestraTemis.

Alvarado, Adolfo. 2007. La prueba judicial - Colección Temas Procesales Conflictivos. Nº 1. Rosario: Juris.

Álvarez, Ernesto. 2009. «El Derecho Constitucional en la formación del abogado». En: Ponencia en el marco del X Congreso Iberoamericano del Derecho Constitucional, organizado por el Instituto Iberoamericano del Derecho Constitucional y la Asociación Peruana de Derecho Constitucional, realizado en Lima, del 16 al 19 de setiembre, Lima.

Asis, Rafael. 1995. Jueces y normas, la decisión judicial desde el ordenamiento. Madrid: Marcial Pons.

Carbonell, Miguel. 2003. Neoconstitucionalismo. Madrid: Trotta.

Castillo, Luis. 2006. Comentarios al Código Procesal Constitucional. Tomo I Título Preliminar y Disposiciones Generales. Lima: Palestra Editores.

Díaz, Javier (Comp). 2004. Textos Constitucionales Históricos. Lima: Palestra Editores.

García, Eduardo. 2006. La constitución como norma y el Tribunal Constitucional. Madrid: Civitas. 
Gascón, Marina y García, Alfonso. 2005. La Argumentación en el Derecho. Lima: Palestra Editores.

Guatiní, Ricardo. 2001. Estudios de Teoría Constitucional. México D.F.: Instituto de Investigaciones Jurídicas de la UNAM.

Landa, Cesar. 2005. Constitución y fuentes del derecho. Lima: Palestra Editores.

López, Luis. 2001. El poder judicial en el Estado Constitucional. Lima: Palestra Editores.

Medina, Manuel. 1996. La vinculación negativa del legislador a los derechos fundamentales. Madrid.: McGraw-Hill.

Montero, Juan. 2008. La prueba en el proceso civil. Barcelona: Civitas.

Nanclares, Andrés. 2004. Los jueces de mármol. Medellín: Señal Editora.

Prieto, Luis. 2005. «Neoconstitucionalismo y Ponderación Judicial». En: Carbonell (ed.). Neoconstitucionalismo(s). Madrid: Trotta. 123-158.

Zagrebelsky, Gustavo. 2005. El Derecho dúctil. Madrid: Trotta.

Fecha de recepción: 12 de marzo de 2015

Fecha de aceptación: 05 de mayo de 2015

\section{Correspondencia}

Luis Robles Trejo

llrobles@hotmail.com 